\title{
Correction to: HoloTube: a low-cost portable 360-degree interactive autostereoscopic display
}

\section{Che-Hao Hsu ${ }^{1} \cdot$ Yi-Leh Wu ${ }^{1} \cdot$ Wen-Huang Cheng ${ }^{2} \cdot$ Yu-Jen Chen ${ }^{3} \cdot$ Kai-Lung Hua $^{1}$}

Published online: 9 December 2021

๑ Springer Science+Business Media, LLC, part of Springer Nature 2021

\section{Correction to: Multimedia Tools and Applications (2017) 76:9099-9132 https://doi.org/10.1007/s11042-016-3502-3}

In the original publication of this article, the given name of the third author was misspelled as "Weng-Huang". The original article has been corrected.

Publisher's note Springer Nature remains neutral with regard to jurisdictional claims in published maps and institutional affiliations.

The original article can be found online at https://doi.org/10.1007/s11042-016-3502-3.

Kai-Lung Hua

hua@mail.ntust.edu.tw

1 Department of Computer Science and Information Engineering, National Taiwan

University of Science and Technology, No. 43, Sec. 4, Keelung Rd., Daan Dist., Taipei 106,

Taiwan Republic of China

2 Research Center for Information Technology Innovation (CITI), Academia Sinica, Taipei, Taiwan Republic of China

3 Department of Radiation Oncology, MacKay Memorial Hospital, Taipei, Taiwan Republic of China 